\title{
Spatially Gain-Tailored Fiber Raman Laser Cladding- Pumped by Multimode Disk Laser at 1030 nm
}

\author{
Yutong Feng, Sheng Zhu, Soonki Hong, Huaiqin Lin, Pranabesh Barua, Jayanta Sahu, Johan Nilsson \\ Optoelectronics Research Centre, University of Southampton, Southampton, SO17 1BJ, UK \\ y.feng@soton.ac.uk
}

\begin{abstract}
A spatially gain-tailored fiber Raman laser with Ge-doped core and P-doped inner cladding generates $46 \mathrm{~W}$ of instantaneous power at $1084 \mathrm{~nm}$ with $64 \%$ slope efficiency when cladding-pumped by a 1030-nm quasi-cw multimode disk laser.

OCIS codes: (140.3510) Lasers, fiber; (140.3550) Lasers, Raman;
\end{abstract}

Multimode-pumping of fiber Raman lasers (FRLs) are the subject of increasing interest in recent years, for wavelength conversion as well as brightness enhancement [1,2]. Often, these are based on germanium-doped multimode graded-index fibers (GIFs) for telecommunication, which are widely available at low cost and offer an unsurpassed combination of loss and Raman gain coefficient, and thus a high figure of merit [3]. However, although GIFs can improve the beam quality as well as the brightness, it has proven difficult to convert a multimode pump beam to a single-mode output beam. For this, double-clad Raman fibers (DCRFs) [4,5] look more promising and have indeed been used to generate single-mode output [6]. Still, single-mode DCRFs need to fulfill several design criteria [7]. Notably, the cladding / core area ratio should not be too large. This limits the size of the inner cladding and thus the scope for power scaling, especially with small single-mode cores. Large core areas are commonly used to improve the power scalability of cladding-pumped fiber laser. The numerical aperture of the core is low to promote single-mode operation. It is relatively easy to fabricate a low-NA large-core DCRF by having similar compositions in core and cladding, e.g., with a pure-silica inner cladding and a germanosilicate core with low Geconcentration [8]. However in this case the Raman gain coefficient becomes similar in the core and the cladding, which makes it difficult to avoid stimulated Raman scattering (SRS) in the cladding.

A possible solution to this is to use different materials in core and cladding, so that the Raman gain coefficient can be different even though the refractive indices are similar. Here, we present a FRL with a germanosilicate core and a phosphosilicate inner cladding, designed to provide higher Raman gain in the core than in the inner cladding. The FRL is cladding-pumped by a quasi-continuous-wave (QCW) multimode disk laser producing 1-ms pulses at $1030 \mathrm{~nm}$ We reach $40 \mathrm{~W}$ of instantaneous Stokes output power at $1084 \mathrm{~nm}$ with $44 \%$ of slope efficiency.

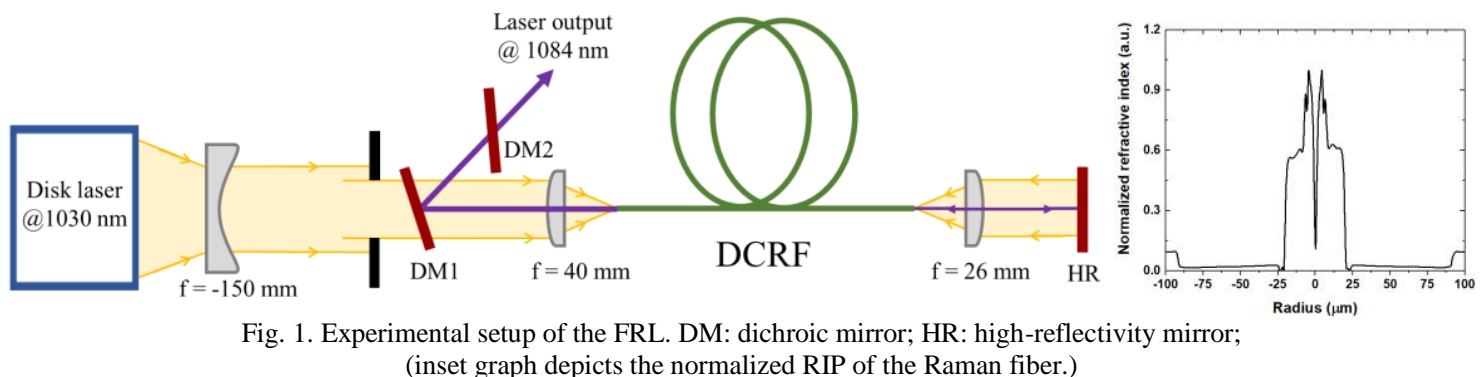

The experimental configuration is shown in Fig. 1. The DCRF was fabricated in-house through modified chemical vapor deposition. It has a protective low-index polymer coating, a pure-silica outer-cladding of $182 \mu \mathrm{m}$ diameter, a phosphosilicate inner cladding of $38 \mu \mathrm{m}$ diameter and $0.15 \mathrm{NA}(\mathrm{V}=17.4$ at $1030 \mathrm{~nm}$ ) with respect to the outer cladding, and a germanosilicate core of $13.5 \mu \mathrm{m}$ diameter and $\sim 0.11 \mathrm{NA}(\mathrm{V}=4.3$ at $1084 \mathrm{~nm})$ with respect to the inner cladding. From the refractive-index profile in Fig. 1, we estimate the $\mathrm{P}_{2} \mathrm{O}_{5}$-concentration to $9 \%$ (mol) and the $\mathrm{GeO}_{2}$-concentration to $10 \%$ (mol). The length used in the experiment is $903 \mathrm{~m}$, with an effective length of $277 \mathrm{~m}$. The total signal round-trip loss is $40.9 \mathrm{~dB}$.

The pump source is a Yb:YAG disk laser (Trumpf HLD 4002) with up to $4 \mathrm{~kW}$ of continuous-wave (cw) or QCW output power at $1030 \mathrm{~nm}$ from a 200- $\mu \mathrm{m}$ diameter, 0.1-NA core delivery fiber connected to a lens arrangement. We collimated the beam to a diameter of $30 \mathrm{~mm}$ and then passed it through an 18-mm aperture, which clips $42 \%$ of the power. This improves the beam parameter product (BPP) from $10 \mathrm{~mm}$ mrad (determined by the delivery fiber) to $6 \mathrm{~mm} \mathrm{mrad}$, so that it is better matched to the $2.9 \mathrm{~mm}$ mrad BPP of the DCRF's inner-cladding. This reduces the pump power not coupled into the inner cladding but into the outer cladding, where it can burn the coating. The pump beam then passes through a dichroic mirror (DM1) with $80 \%$ transmission for the pump beam and $92 \%$ reflection for the Stokes beam at $1084 \mathrm{~nm}$ and is then launched into the Raman gain fiber through an $\mathrm{f}=40$ 
$\mathrm{mm}$ aspheric lens. The overall pump launch efficiency is $9 \%$ from the disk laser into the inner cladding. The fiber has perpendicular cleaves in both ends. The fiber facet in the pump launch end serves as a 4\%-reflecting output coupler for the laser cavity, while DM1 deflects the generated Stokes beam from the path of the pump beam. A second dichroic mirror, DM2, cleans the Stokes beam from any residual pump light. In the far end of the fiber, a collimating $\mathrm{f}=26 \mathrm{~mm}$ aspheric lens and a high-reflectivity (HR) mirror with reflectivity $>99 \%$ for pump and signal close the laser cavity. The mirror also reflects escaped pump back into the fiber in order to double-pass the pump.
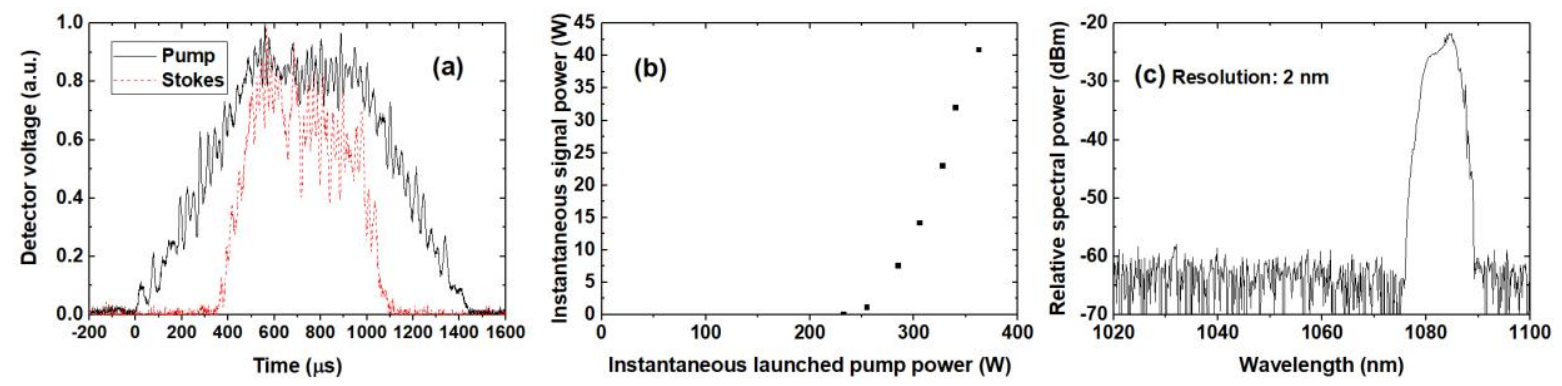

Fig. 2. (a) Temporal traces of pump and Stokes pulses at maximum power (b) instantaneous signal power $v s$. instantaneous pump power launched into the inner cladding; (c) laser output spectrum at maximum power following spectral filtering in DM2.;

Despite these protective steps, the fiber still failed at the pump launch under cw operation. Therefore, we set the pump source to operate at $10 \mathrm{~Hz}$ with $1 \%$ duty cycle. This reduces the thermal load. Fig. 2 (a) shows the temporal traces of the pump and Stokes pulses at maximum pump power, which was $28 \mathrm{~W}$ (average) and $362 \mathrm{~W}$ (instantaneous) launched into the inner cladding. The pump pulse consists of a leading ramp, a plateau, and a trailing

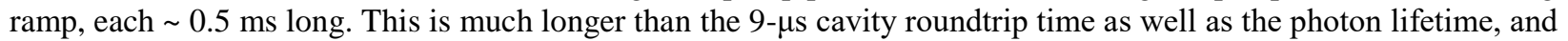
since SRS is in itself nearly instantaneous, the FRL can be considered to operate in a quasi-steady-state. Therefore, we consider the instantaneous power as averaged over the central 0.5-ms. Fig. 2 (b) shows the result. The Stokes power is measured after DM2 but plotted as the value before DM1. The threshold becomes $250 \mathrm{~W}$, which agrees with simple estimates. The Stokes power then grows with $44 \%$ slope efficiency to $40 \mathrm{~W}$ at full power. A higher slope efficiency of $64 \%$ is achieved by cutting the fiber to $703 \mathrm{~m}$ to reach a maximum Stokes power of $46 \mathrm{~W}$. Further characterization of this and shorter lengths is in progress.

The beam quality was measured to $\mathrm{M}^{2}=4.8$ at full power. This is higher than expected for a core $\mathrm{V}$-value of 4.3 and indicates SRS in the inner cladding. Fig. 2 (c) shows the spectrum measured after DM2 at full power. The output peaks at $1084 \mathrm{~nm}$, for a Raman shift of $484 \mathrm{~cm}^{-1}$. This is larger than the germanosilicate value of $\sim 440 \mathrm{~cm}^{-1}$ [9], and is closer to that of phosphosilicate [10]. This also indicates there is SRS in the inner cladding, or that P has diffused into the core. In the absence of diffusion, we estimate the Raman gain coefficient for unpolarized light to $1.03 \times 10^{-13} \mathrm{~m} / \mathrm{W}$ in the core (for germanosilicate [9]), and to $0.88 \times 10^{-13} \mathrm{~m} / \mathrm{W}$ in the inner cladding (for phosphosilicate, from [10]). This small difference suggests that other dopants such as $\mathrm{Al}_{2} \mathrm{O}_{3}$ may be better.

This work was funded by AFOSR (FA9550-15-1-0041). The data for this paper is openly accessible at http://doi.org/10.5258/SOTON/D0367.

\section{References}

[1] Ekaterina A. Zlobina, Sergey I. Kablukov, Alexey A. Wolf, Ilya N. Nemov, Alexander V. Dostovalov, Valentin A. Tyrtyshnyy, Daniil V. Myasnikov, and Sergey A. Babin, "Generating high-quality beam in a multimode LD-pumped all-fiber Raman laser," Opt. Express 25, 12581-12587 (2017).

[2] Yaakov Glick, Viktor Fromzel, Jun Zhang, Nikolay Ter-Gabrielyan, and Mark Dubinskii, "High-efficiency, 154 W CW, diode-pumped Raman fiber laser with brightness enhancement," Appl. Opt. 56, B97-B102 (2017).

[3] T. Yao, A. Harish, J. Sahu, and J. Nilsson, "High-Power Continuous-Wave Directly-Diode-Pumped Fiber Raman Lasers," Appl. Sci. 5, 1323-1336 (2015.)

[4] C. A. Codemard, P. Dupriez, Y. Jeong, J. K. Sahu, M. Ibsen, and J. Nilsson, "High-power continuous-wave cladding-pumped Raman fiber laser," Opt. Lett. 31, 2290-2292 (2006).

[5] C. A. Codemard, J. Ji, J. K. Sahu, and J. Nilsson, "100 W CW cladding-pumped Raman fiber laser at 1120 nm”, in Fiber lasers VII: technology, systems, and applications, (Institute of Electrical and Electronics Engineers, New York, 2010) pp. 75801N-75801N-7

[6] J. Nilsson, J. K. Sahu, J. N. Jang, R. Selvas, D. C. Hanna, and A. B. Grudinin, "Cladding-pumped Raman fiber amplifier," in Optical Amplifiers and Their Applications, J. Nagel, S. Namiki, and L. Spiekman, eds., Vol. 77 of OSA Trends in Optics and Photonics Series (Optical Society of America, 2002), paper PD2.

[7] J. Ji, C. A. Codemard, J. K. Sahu, and J. Nilsson, "Design, performance, and limitations of fibers for cladding-pumped Raman lasers," Opt. Fiber Technol. 16, 428-441 (2010).

[8] J. Ji, C. A. Codemard, A. Boyland, J. K. Sahu, and J. Nilsson, "Beam Quality and Spectral Evolution in Large-Core Cladding-Pumped Cascaded-Raman Fiber Converter," in Lasers, Sources and Related Photonic Devices, (Optical Society of America, 2010 ), paper AMB3.

[9] S. T. Davey, D. L. Williams, B. J. Ainslie, W. J. M. Rothwell, and B. Wakefield, "Optical gain spectrum of $\mathrm{GeO}_{2}-\mathrm{SiO}_{2} \mathrm{Raman}$ fibre amplifiers," IEE Proc. J - Optoelectron. 136, 301-306 (1989).

[10]Evgeny M. Dianov, "Advances in Raman Fibers," J. Lightwave Technol. 20, 1457-1462 (2002). 